\title{
New Infrastructure of NGN and IMS Networks
}

\author{
Saeed Mahdavi, Maryam Sabet Sarvestani, and Mohammad Doosti
}

\begin{abstract}
This paper is about the introduction of new generation networks and include network standard used this type of structure is providing services(vas).The infrastructure requirements in the fixed network and how network communications convergance fixed and mobile network. And the use of virtual IP and virtual network IMS to provide better customer service offered.
\end{abstract}

Index Terms-IMS, CSCF, subsystem, 3GPP, network.

\section{INTRODUCTION}

IMS started as a technology for $3^{\text {rd }}$ Generation mobile networks (under the auspices of the $3^{\text {rd }}$ Generation Partnership Project (3GPP), but it is now spreading to next generation wireline networks and is going to be a key to Fixed Mobile Convergence (FMC). It builds upon Session Initiation Protocol (SIP) which has emerged as the crucial technology for controlling communications in IP-based next generation networks (NGN).

IMS is about services and applications, enabling service providers to offer rich multimedia services across wireless, packet and traditional circuit switched networks. It is standards based and uses open interfaces and functional components that can be assembled flexibly into hardware and software systems to support real-time interactive services and applications. The basic set of standards for IMS implementation were released in 2004 and the first implementations are beginning in the European wireless markets. The standards organizations are heavily involved in developing standards to fill inevitable gaps and to add new capabilities. However IMS is still untested in real-life major carrier networks and its wide scale implementation is some years away. That being said, it is the most likely evolution path for next generation networks, including those for Emergency Services. This paper will illustrate the current standards relating to IMS, define IMS and the show the functional elements that comprise it. It will explain how elements interact and illustrate example call flows. Not much work has begun on relating Emergency Services to IMS. This paper will describe what has been done in $3 \mathrm{GPP}$ and highlight areas where additional attention is required [1].

Manuscript received March 12, 2016; revised June 13, 2016.

Saeed Mahdavi is with Headquarters of Fars Province Telecom (TCF), Shiraz, Iran (e-mail: mahdavisa@gmail.com).

Maryam Sabet Sarvestani is with Information Technology Department, Paramedical Sciences School, Shiraz University of Medical Sciences, Shiraz, Iran (e-mail: m.sabet@aut.ac.ir).

Mohammad Doosti is with Institute of Applied Science Technology, Jahad Deneshgahi (IASTJD), Shiraz, Iran (e-mail: moh.doosti@gmail.com).

\section{OVERALl ARChITECTURE}

The TISPAN NGN functional architecture ES 282001 is structured according to a service layer and an IP-based transport layer (see Fig. 1 below).The service layer comprises the following components:

- The IP Multimedia Subsystem core component (IMS).

- The PSTN/ISDN Emulation Subsystem (PES).

- Other multimedia subsystems and applications.

- Common components (i.e. used by several subsystems) such as those required for accessing applications, charging functions, user profile management, security management, routing data bases (e.g. ENUM), etc.

IP-connectivity is provided to NGN user equipment by the transport layer, under the control of the Network Attachment SubSystem (NASS) and the Resource and Admission Control Subsystem (RACS). These subsystems hide the transport technology used in access and core networks below the IP layer. The present document further describes the functional architecture of the IP Multimedia Subsystem (IMS) core component [2].

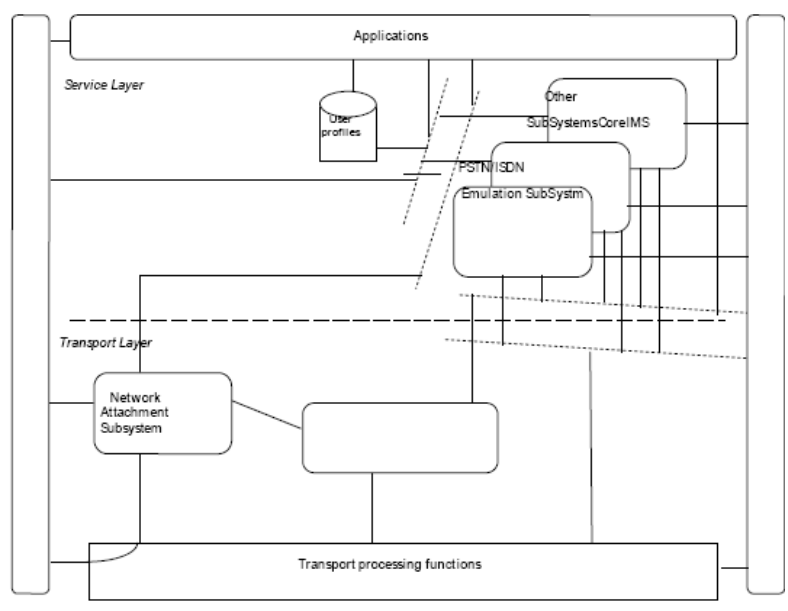

Fig. 1. TISPAN overall architecture.

\section{OVERVIEW}

The NGN IP Multimedia Subsystem (IMS) supports the provision of SIP-based multimedia services to NGN terminals. It also supports the provision of PSTN/ISDN simulation services.

Functional entities of an IMS may be used by an operator in support of transit network scenarios. The routing may be performed, depending on the entity performing the routing, and depending on the traffic case, on signaling information, configuration data, and/or data base lookup.

The NGN IMS, also known as "Core IMS" is a subset of the 3GPP IMS defined in TS 123002 which is restricted to the session control functionalities. Application Servers (AS) and 
transport/media related functions such as the Multimedia Resource Function Processor function (MRFP) and the IP Multimedia Gateway Functions (IM-MGW) are considered to be outside the "core IMS".

Although essentially identical to the 3GPP IMS entities, NGN IMS functional entities might exhibit minor variations in behavior, due to differences in access networks and user equipment. However, the NGN IMS architecture defined in the present document remains compatible with 3GPP-defined IP-connectivity access networks (IP-CAN) and as such can provide services to user equipment connected to both fixed broadband access and 3GPP IP-CANs.

Fig. 2 below; illustrates the position of the IMS in the overall NGN architecture. The IMS interfaces the following components:

- User Equipment.

- The Resource and Admission Control Subsystem.

- The Network Attachment Subsystem.

- The PSTN/ISDN.

- The PSTN/ISDN Emulation Subsystem.

- Other multimedia subsystems.

- Charging Functions.

- Network Management Functions.

- Applications and other common architectural elements.

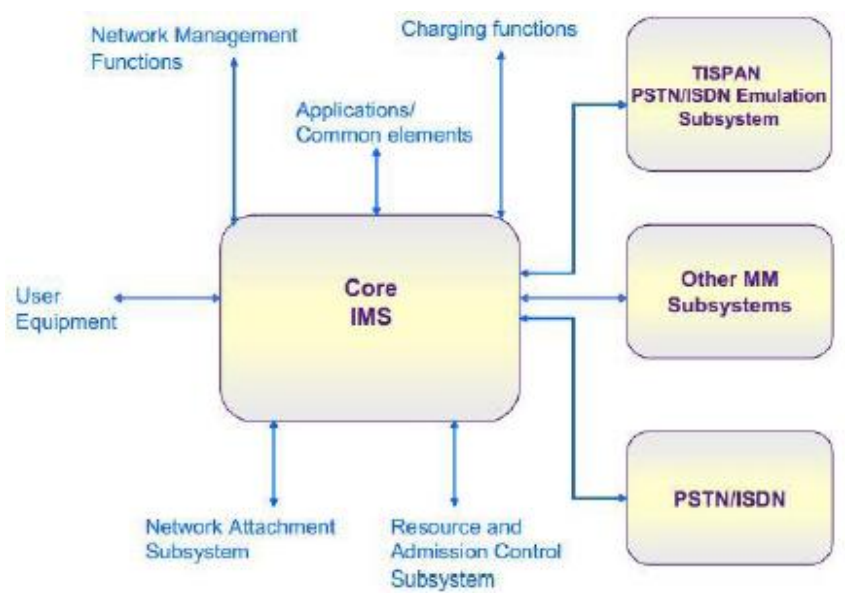

Fig. 2. TISPAN IMS and its environment.

\section{DEFINITIONS OF IMS}

- IMS : IP MultiMedia Subsystem

- 3GPP defined communication platform for multi-media session/service control based on SIP protocol.

- Over All-IP network.

- Core Network for unified service over Fixed and Mobile network

- IMS was born while 3GPP make standard for 3G Wireless network with detailed functions and interfaces

- IMS defines the functional architecture \& interfaces of service network Compliance with International standard make it easy to introduce new service or interwork with other network equipment.[2]

\section{3GPP ARCHITECTURE}

- Defined components for session control based on IP

- Assumes Wireless network as the access network
- $3 \mathrm{GPP}$ is the organization from mobile society

- But, every components except PCRF which controls network resource has no direct dependency with the network.

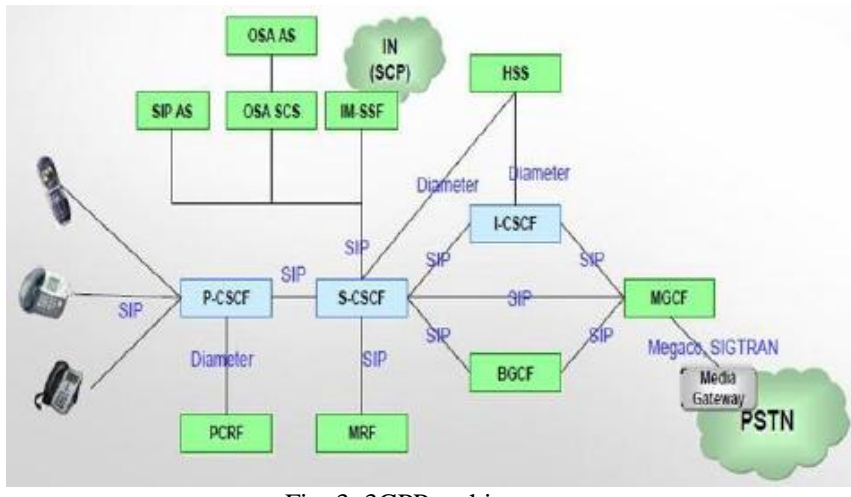

Fig. 3. 3GPP architecture

- IMS is defined as a network architecture that defines functional elements. Each functional element does not have to relate one-to-one to a physical element. A number of functional elements can be incorporated into a physical element depending upon a vendor's implementation. The Service Architecture defines standard methods for services to be introduced while the Core Network defines the interactions between functional elements.

- IMS is multimedia. Therefore you need to think beyond a typical voice call. The context could be voice, video or graphics; or a combination correlated between two or more parties [3].

- IMS begin with 3GPP and ETSI, both which have their roots in Europe. To introduce IMS in the U.S. there are some adoptions that will be needed to accommodate U.S. nuances. It is likely that these will be handled by TIA and ATIS.

- IMS relies heavily existing standards developed by the IETF. Organizations representing IMS are also heavily influencing the IETF activity to evolve or develop new standards needed to complete the IMS picture.

- FMC - Fixed Mobile Convergence is the new buzz word of the industry. IMS's intended applicability is across wireless, wireline, cable, enterprise and other networks.

- One of the major attributes, which may have more play in wireless, is the idea of service transparency no matter what network you are in (e.g. the home or visited network).

- There is a recognition that IMS must evolve into the networks and that there needs to be interworking between existing functionality and the newly envisioned capabilities.

- Media Gateways are the interface between IMS and the legacy PSTN world. This allows calling between the richly featured multimedia IMS environment to the existing voice networks.

- The interworking between legacy endpoints and the IMS network allow those endpoints to access the features and functions that IMS can provide. Of course, this functionality may be limited by the capabilities of the endpoint. For example, network based features may be 
available to the legacy endpoint, but not multimedia.

- IMS defines common support for Classes of Service, Quality of Service, security and other attributes. However, standards are still evolving in many of these areas.

- Since IMS is built upon IP, it supports the flexibility and scalability to support mobility, portability, service creation, etc. All of these together may provide operational and financial improvements beyond existing networks.

- IMS defines how to develop subscriber databases which include User Profiles that enumerate identity, services, security levels, etc.

- IMS defines common session control that applies to any media. So the way a voice call is set up is identical to how a video call is set up. Based upon the class of service, different resources may be allocated in the network.

- IMS specifies common OAM\&P environment that allows the evolution of operation support systems.

- IMS, in its self, does not define services. However, it defines how services are accessed. These services may be inherent in the IMS network or can provide gateways to existing service platforms.

- IMS is designed to be applicable to the evolution of all types of networks. The major wireless carriers have committed to IMS as their next generations network. All of the U.S. major wireline carriers have embraced IMS in their evolution path. Cable network companies have not embraced IMS as of yet since Cable Labs has just defined IP-based network topology that was pre-IMS. It is thought that as cable networks evolve, they too will embrace IMS.

- One major advantage of IMS's commonality is that carriers from each discipline can purchase equipment based upon the same standards, thereby potentially decreasing the cost to provide duplicate

There are a number of organizations whose activities directly apply in defining IMS. The initial concept came from 3GPP in Europe to be the evolution of GSM networks. 3GPP depended heavily upon the work of IETF in defining SIP and related protocols. It became clear that IMS had broader appeal and organizations such as TISPAN began defining extensions needed for the wireline network.

IMS is being defined in phases. The early phases focused specifically on GSM evolution. Releases kept building features and functionality and expanding the scope of applicability beyond GSM. Release 12 is to incorporate Emergency Services (see Fig. 4 and Fig. 5).

It is important that the U. S. Emergency Services Industry be involved in this effort to assure its needs are recognized. While IMS has global scope, the needs of Emergency Services in Europe are different that those in the U.S. A common and coordinated approach to Emergency Services will benefit the global community.

IMS is designed to be applicable to the evolution of all types of networks. The major wireless carriers have committed to IMS as their next generations network. All of the U.S. major wireline carriers have embraced IMS in their evolution path. Cable network companies have not embraced IMS as of yet since Cable Labs has just defined IP-based network [4].

IMS is being defined as the convergence vehicle for all types of access connectivity. IMS allows typical Centrex customers to migrate to IP-based Centrex services and provides direct connectivity from the myriad of IP PBXs that are being deployed. IMS provides a natural evolution as LECs deploy their broadband access to the home as well as providing cable providers a standardized approach for network evolution. This theme extends to the introduction of WiFi or WiMax technologies that are inherently IP based.

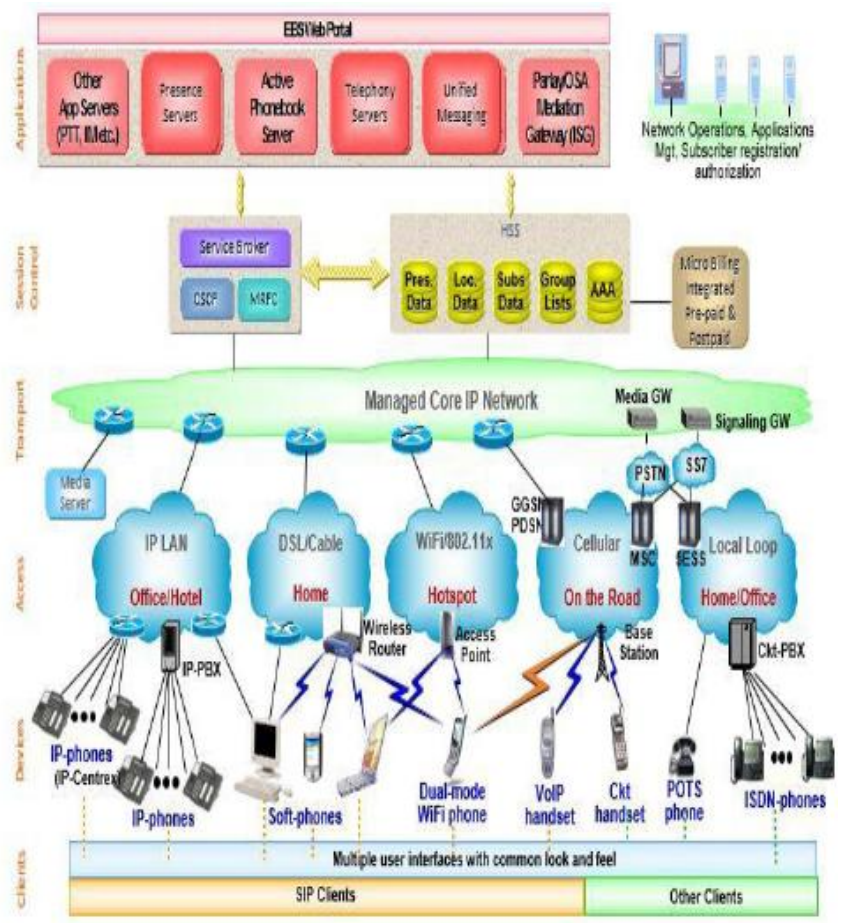

Fig. 4. IMS converged communications services vision.

It is recognized that not all end points will be SIP enabled. Therefore, legacy systems can take advantage of the IMS services by entering the network through signaling and media gateways.

As shown in the wireless cloud, a network can evolve such that it can take advantage of existing access techniques while evolving to IP connectivity that can natively interconnect to the IMS core.

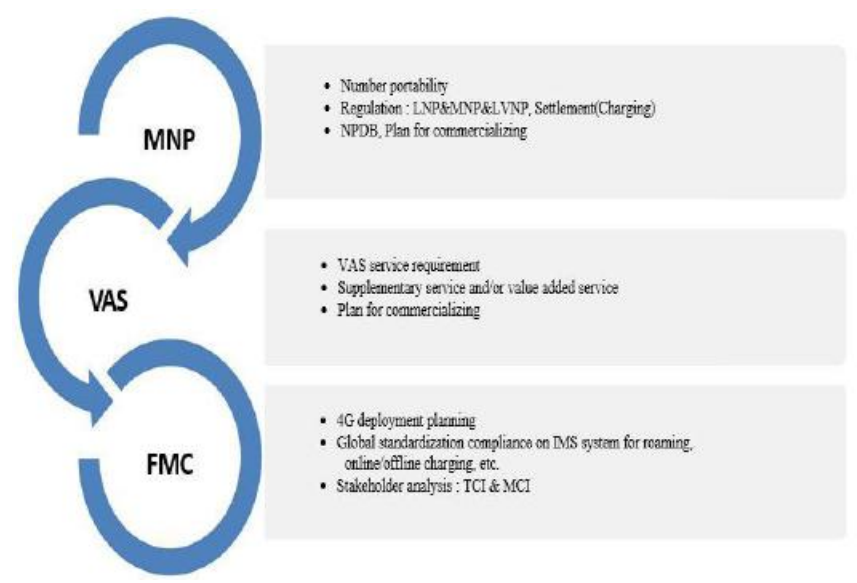

Fig. 5. Checkpoint for additional requirements.

VI. VAlue AdDED SERVICES ARChitecture (VAS) 


\section{A. Overview}

Three types of Application Server Functions (ASF) can be accessed by the IMS through the ISC or Ma reference point.

\section{B. IP Application Servers (SIP AS)}

- The IM-SSF Application Server.

- The OSA SCS Application Server.

A SIP Application Server may contain "Service Capability Interaction Manager" (SCIM) functionality and other application servers. The SCIM functionality is an application which performs the role of interaction management. The internal structure of the application server is outside the standards (see Fig. 6).

The purpose of the IM SSF is to enable access to IN service logic programs hosted in legacy SCPs. The IM-SSF functionality encompasses the emulation of the IN Call Model (BCSM) on top of SIP signaling, IN triggering and feature management mechanisms, emulation of the IN Service Switching Finite State Machine and interworking with INAP. The purpose of the OSA Service Capability Server is to provide access to OSA applications, according to the OSA/Parlay framework ES 201 915-1.

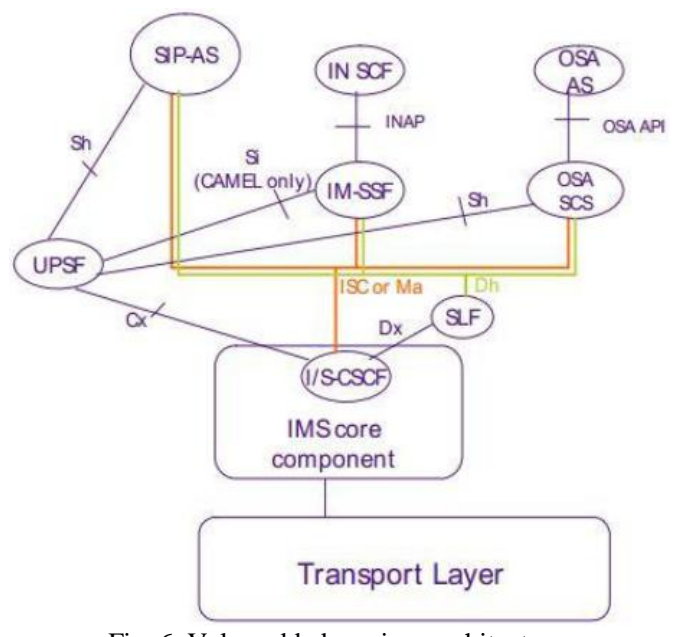

Fig. 6. Value added services architecture.

The Service-CSCF to AS reference point is used to forward SIP requests, based on filter criteria associated with the originating or destination user.

The Interrogating-CSCF to AS reference point is used to forward SIP requests destined to a Public Service Identity hosted by the AS directly to that AS.

\section{NeW VAlue AdDED SeRVice (VAS)}

\section{A. Overview}

According to IMS networks developments and applications require more, more value-added services (VAS) for a variety of activities, it is necessary to add some new services to this structure, since a wide group of organizations and companies are member of this network, it seems the leading service with its' capabilities is very popular (see Fig. 7).

\section{B. Example of New Service}

Generally all organizations from medium-sized companies to large use their own corporate portals and corporate email, most of corporate portals were Desktop based in the past, but today there is a word called Cloud that made companies to develop their software by this structure.

Now, our new service suggestion is "Cloud Corporative Portal" that explains in the following:

By this service all managers and their employees are able to check \& do their daily works in anywhere, anytime and by any devices. So, for doing this just you need to register in IMS network and have only one device such as Laptop, Tablet, Smart Phone and etc....

We should pay attention to the following points for implementation:

1) A Data Center for maintain and lunch servers

2) Suitable servers depends on Benchmark

3) Implement corporate portal compatible with Cloud standard

4) Necessary Benchmark

5) Compatible portal with a variety of devices (laptop, tablet, smartphone, etc.)

6) Data encryption

7) SLA agreements

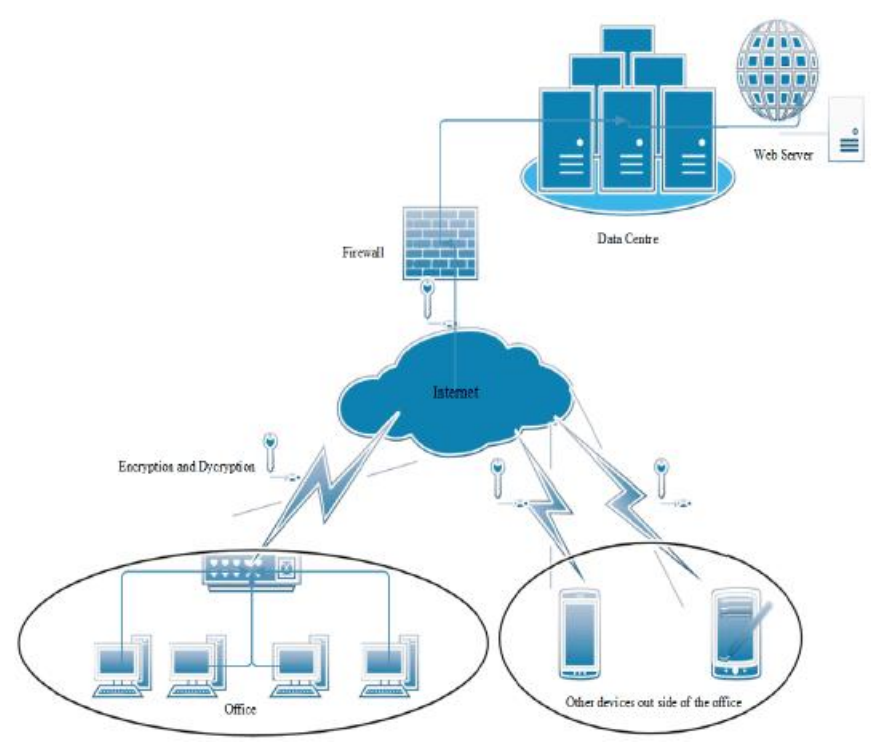

Fig. 7. Cloud corporative.

\section{BROADCASTING CONVERGENCE INTO IP-BASED NETWORKS}

The digitalization of content, added to the shift towards IP-based networks, the diffusion of high- speed broadband access, and the availability of multi-media devices, allowed an increasing convergence of broadcasting and telecommunication sectors. The production and diffusion of audio- visual content does not seem to be limited to traditional broadcasters anymore. Telecommunication operators are providing content along with Internet access, newly emerging providers are offering access to content over IP, and traditional broadcasters are crossing over to other platforms, transmitting their programs also over IP networks.

Furthermore, the development of next generation mobile services-using $3 \mathrm{G}$ and $4 \mathrm{G}$ networks, or mobile broadcasting systems - enables the delivery of high quality audiovisual (AV) content to portable devices and mobile phones. Convergence is nowadays a reality, with different types of content and communication services delivered through the 
same pipes and consumed over a variety of platforms and user devices. Convergence over multiple access platforms has not only affected the distribution market, but also created new forms of usage, providing consumers with greater choice and control over content. Multimedia, interactive audiovisual services are increasingly transforming users from passive watchers of TV programs to active players able to decide what they want to see, when and on which device. Video on Demand, Personal Video Recorder (PVR) services, peer to peer (P2P), or user-created video, therefore, herald an important change in the traditional broadcast model to exchange audiovisual content among large audiences. Media consumption, tastes and preferences may become more fragmented, the importance of social networks as a means to participate in content creation will probably continue to grow, and there will be an increasing demand for new types of content, able to fully capture the new capacity of the Internet for interactivity, non-linear consumption and participation.

The evolution of technology does not necessarily change many of the social and cultural broadcasting policy objectives, but technology may change the way that they are presently implemented and may allow for increased market liberalization than that which has been common in the sector whileallowing the core policies to be maintained. The digitalization of transmission, for example, enables a more efficient use of spectrum than analogue transmission, increasing significantly the number of terrestrial broadcasting channels which can be made available. When analog TV signals are switched off, a significant amount of spectrum bandwidth will be freed up, and will be available potentially for other applications, such as mobile television, high-definition television, mobile broadband networks and WiMAX networks. Audio-visual content providers may include network operators, which are usually provide digital television and content over IP networks as part of their "triple play" bundles, or new service providers, such as Joost, using $\mathrm{P} 2 \mathrm{P}$ technologies to stream content over the Internet, or YouTube, based inter alia on user created content. Broadcasters are also entering the IP market, launching new content platforms, such as Hulu-a NBC/NewsCorp venture

As the market for audiovisual services becomes more dynamic, content producers will be able to offer services directly to all new markets without intermediaries or gatekeepers. With content available on new platforms and networks, there should be lower entry barriers, and the sector could become more open and competitive over the next years. At the same time this will bring up the issue of the need for network neutral policy approaches, for both fixed and mobile networks, in order to avoid the creation of barriers to access for independent service providers

In addition, existing government instruments to control broadcasting content - such as quotas for protection of language and culture, pluralism requirements, or must carry obligations-are challenged by the new multiplatform environment, and may need to be adjusted in order to continue to fulfill their goals.

Convergence not only leads to a larger and more competitive market, but also a more international market. A globally structured market - in terms of ownership, investment, and distribution and marketing strategies - offers an enormous potential to the media industries, but also poses new challenges to national regulation, which may not always be compatible across borders, therefore risking to be less effective, not enforceable, or - if excessively restrictive - to slow down growth of media players in an international content market.

\section{A. Virtualizing the Core IP Network}

\section{1) Overview}

The cloud computing is a culmination of numerous attempts at large scale computing with seamless access to virtually limitless resources. Cloud computing is an internet-based computing, whereby shared resources, software and information are provided to computers and other devices on-demand, like the electricity grid.

\section{2) Description}

To be able to guarantees QoS and Real Time parameters in a cloud network, and also to achieve goals such as flexibility, storage capacity and bandwidth we use virtualization that enable us to make changes basis of our needed. In this structure depends on our necessry, we can change capacity and bandwidth by increasing or decreasing VMs or turning them on or off. It is important to note that these items have a very great impact on cost efficiency.

As shown in this structure, each VDC network is connected to both VNF that is responsible redundancy operations and also each VDC manage the VNFs (see Fig. 8).

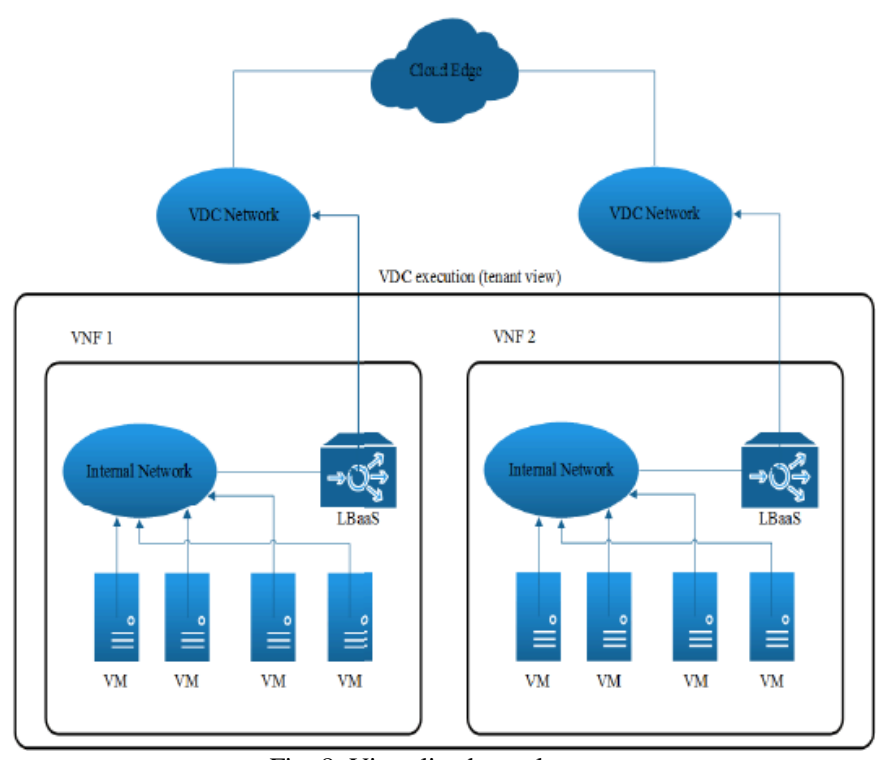

Fig. 8. Virtualized core layer.

\section{IMS CLOUD ARCHITECTURE}

Cloud computing is changing the way of developing, deploying and managing applications. However, as typical Internet-based applications, cloud computing services lack carrier-grade signaling control mechanism and cannot guaranty Quality of Service (QoS), which have actually become technical barriers for telecom operator to provide commercial public cloud services. On the other hand, as the core signaling architecture of Next Generation Networking (NGN), IP Multimedia Subsystem (IMS) is facing the problem of the lack of innovative value-added services. This page presents an architecture to support cloud computing 
services over IMS. In the proposed architecture, cloud services are regarded as the general IMS applications and then cloud clients are allowed to access cloud services under the control of Session Initiation Protocol (SIP) signaling and QoS mechanism of IMS. This page introduces architecture overview and cloud service relevant functional components, and mainly discusses several key issues including cloud notification mechanism, QoS and charging control of IMS-based cloud computing services (see Fig. 9 and Fig. 10).

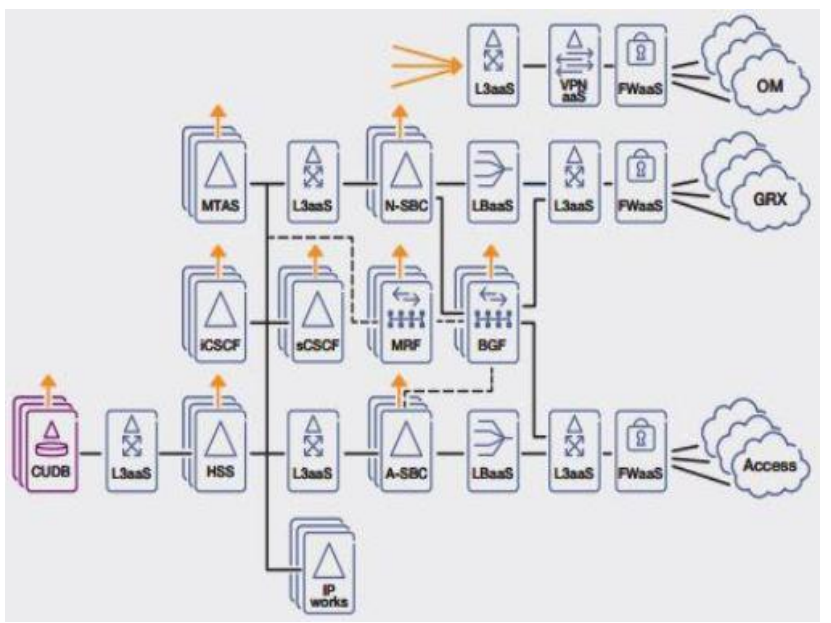

Fig. 9. IMS core network.

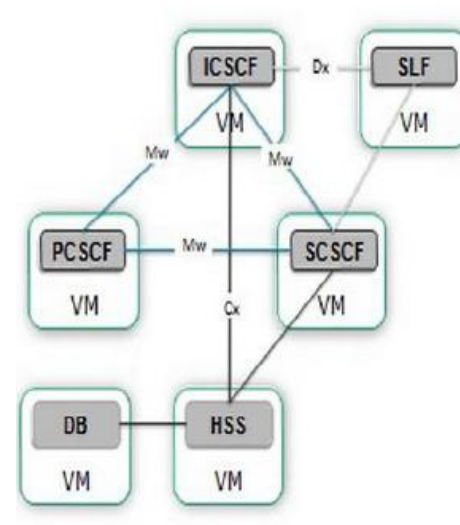

Different load balancing mechanisms for different components

- DNS load balancing between: UE andPCSCF PCSCF and ICSCF

- SLF between:

ICSCF and HSS

SCSCF and HSS

- Capabilties set between: ICSCF and SCSCF

Each time an instance of a network element is created/ deleted. the Orchestrator has to update the different load balancers

Needed an API to dynamically change the topology

Fig. 10. NFV-based architecture virtualized-IMS (v-IMS).

\section{FiX MobiLe CONVERGEnce}

In the future network technology such as IMS (IP Multimedia Subsystem), should provide a standardized next generation architecture based on Internet Protocol (IP) for operators, and allow for the provision of mobile and fixed services using converged handsets embedding a radio interface such as cellular/Wi-Fi or cellular/Bluetooth dual-mode handsets. Currently, the main factor promoting FMC is the trend towards VoIP-enabled wireless telephony (VoWi-Fi), i.e. devices that use Wi-Fi to connect to a VoIP service such as Skype or roam between cellular and wireless LAN systems. Someof the VoWi-Fi operators are at present providing $\mathrm{Wi}-\mathrm{Fi}$ based only services, but some are starting to offer FMC services by combining cellular services with VoWi-Fi. Challenges to mobile telecommunications operators are also coming from Wi-Fi hotspot operators, such as Boingo, allied with Skype. Some mobile operators are linking or considering linking their cellular networks with Wi-Fi hotspots and using VoWi-Fi to improve indoor coverage and offer low-cost calling in Wi-Fi locations. At present there various ways being used to provide FMC services, some of which are more technologically integrated than others. Dual-mode cellular/Wi-Fi handsets and using $\mathrm{Wi}-\mathrm{Fi}$ modems in the home environment to access VoIP through ADSL connections can be found in some countries. There are less evolved forms of FMC using cellular/Wi-Fi dual-mode handsets that do not have a handover function or have a handover function but do not utilize a fixed voice or broadband network in the home. Services also exist linking both fixed and mobile networks which are not technologically converged, such as those offering a single voice mailbox over both fixed and mobile networks.

The deployment of NGN is expected to accelerate the offer of FMC services which are seamless to the user and use least cost routing. In turn, this may require that regulators review existing frameworks to ensure that they are not a disincentive to the development of new services, and that existing frameworks treat new services in a technologically neutral way. Numbering policies also have to accommodate FMC services and, if existing geographic numbers are used, then, in a calling party pays system, it may be necessary to devise ways to inform the call originator if different charges will be assessed based on the called party's location. It may also be important for regulators to develop adequate market tests given that the incumbents already have market power and often their mobile operators are also the market leaders; the development of FMC can augment this market power (see Fig. 11-Fig. 13).

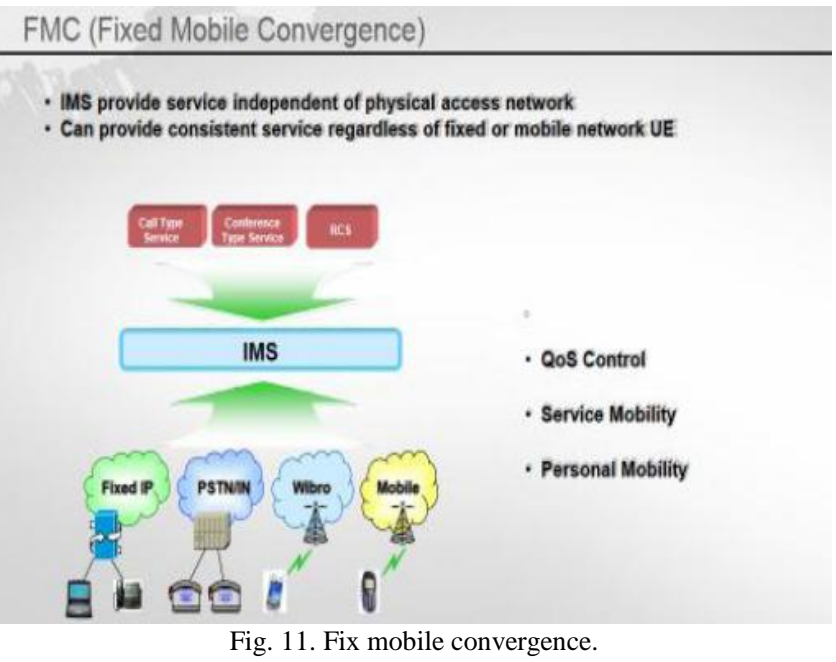

- IMS, FMC platform deployed in All-IP network

- Expand customer adding to All IP based subscribers over IMS

- Developing Converged IMS platform in accordance with global standard [5].

\section{INTEGRATED SECURITY IMS/FMC SOLUTION}

1) User Authentication

2) Network Protection

3) Security Architecture 
A. User Authentication

- Http Digest (RFC 2617)

- Improved Http Digest (OMA-PoC)

- Early IMS (3GPP TR 33.978)

- IMS AKA (3GPP TS 33.203, R5)

- NIBA (TISPAN ES 283 003)

- IMS AKA (R7, Fixed Access Extension)

B. Network Protection

- Network Domain Security / Inter-operator Interface

- SPIT Prevention

- Hardening

- Security Tests and Scans

- SECANT

- Security Services Add-on

- IMS Network Domain Security / Inter-operator Interface

- Security Gateways (SEG) provide secure tunnels between networks

- Integrity and confidentiality protection between SEGs

- State-of-the-art Firewalls for IMS protection

- Traffic between subnets is controlled by access control lists on routers or firewalls or by proxies

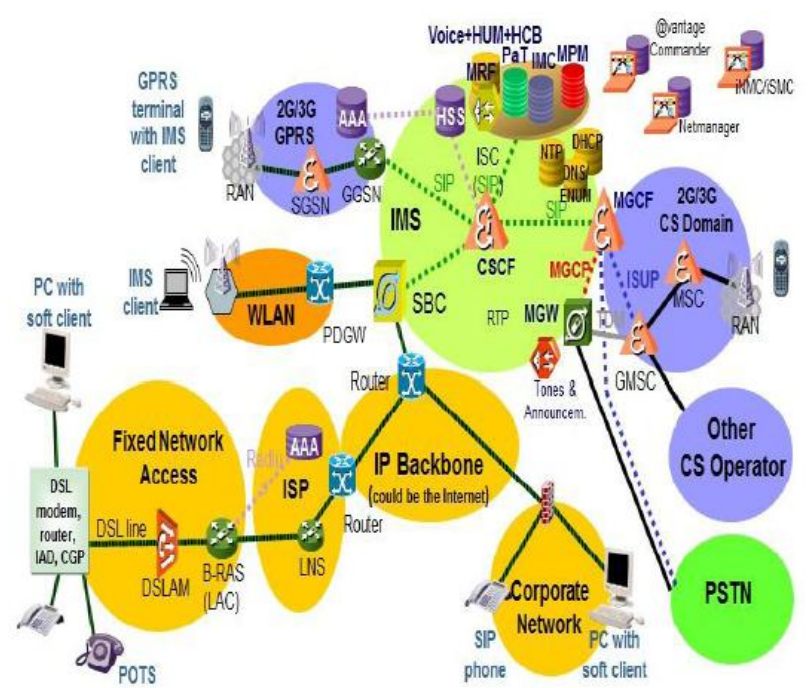

Fig. 12. Fix mobile.

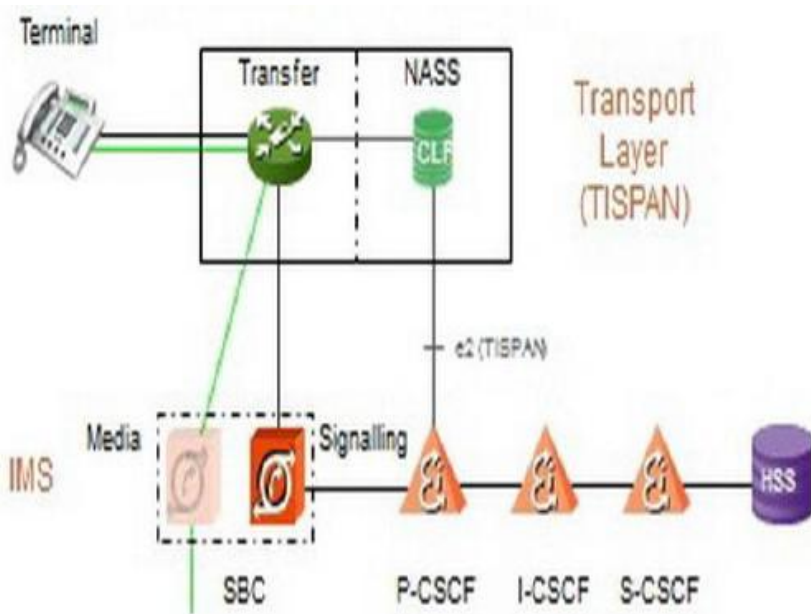

Fig. 13. Transport layer.

\section{Security Architecture}

- IMS/FMC subnets:

- IMS/FMC Control Network
- $\mathrm{k}$ for SIP and other signaling flows

- Media Network for RTP media flows

- Web based services portals

- Management network for OAM traffic

- Installation, Backup \& Restore Network

- Further separation possible (e.g. for Application Servers, HSS, etc. [6].

\section{NIBA AUTHENTICATION}

- NASS IMS Bundled Authentication

- Reuse of authentication in fixed access network for IMS

- NASS: Network Attachment Sub-System

- CLF: Connectivity Session Location and Repository Function

- SBC: Session Border Controller

APPENDIX

ABBREVIATIONS

\begin{tabular}{|l|l|}
\hline AF & Application Function \\
\hline AS & Application Server \\
\hline ASF & Application Server Function \\
\hline BCSM & Basic Call State Model \\
\hline BGF & Border Gateway Function \\
\hline CAMEL & Customized Application for Mobile Enhanced Logic \\
\hline CCBS & Call Control on Busy Subscriber \\
\hline CSCF & Call Session Control Function \\
\hline E-CSCF & Emergency-Call Session Control Function \\
\hline IBCF & Intermediate Breakout Control Function \\
\hline I-BGF & Interconnection-Border Gateway Function \\
\hline I-CSCF & Interrogating-Call Session Control Function \\
\hline IM-MGW & IP Multimedia - Media GateWay \\
\hline IMS & IP Multimedia Subsystem \\
\hline IN & Intelligent Network \\
\hline INAP & IN Application Part \\
\hline IN-SCF & Intelligent Network Switching Control Function \\
\hline IP-CAN & IP-Connectivity Access Network \\
\hline ISDN & Integrated Services Digital Network \\
\hline MGCF & Media Gateway Control Function \\
\hline MGF & Media Gateway Function \\
\hline MM & MultiMedia \\
\hline MRFC & Multimedia Resource Function Controller \\
\hline MRFP & Multimedia Resource Function Processor \\
\hline NAPT & Network Address and Port Translation \\
\hline NASS & Network Attachment SubSystem \\
\hline NGN & Next Generation Network \\
\hline OSA & Open Service Access \\
\hline P-CSCF & Proxy-Call Session Control Function \\
\hline PDF & Policy Decision Function \\
\hline PES & PSTN/ISDN Emulation Subsystem \\
\hline & \\
\hline
\end{tabular}

\section{REFERENCE}

[1] ETSI ES 282 001: "Telecommunications and internet converged services and protocols for advanced networking (TISPAN)," NGN Functional Architecture.

[2] ETSI ES 282010 , "Telecommunications and internet converged services and protocols for advanced networking (TISPAN)," Charging [Endorsement of 3GPP TS 32.240 v6.3.0.

[3] ATIS NGNFG, Next Generation Network (NGN) Framework, Part II: NGN Roadmap 2005, Next Generation Network Focus Group, Technology and Operations Council (TOPS), Alliance for Telecommunications Industry Solutions (ATIS), Issue 1.0.

[4] ATIS_Framework. [Online]. Available: http://www.atis.org/topsc/Docs/ATIS_Framework_Part_2_NGN_Roa dmap_FINAL.pdf

[5] DMS_PUB. [Online]. Available: https://www.itu.int/dms_pub/itu-t/oth/06/5B/T065B0000140013PDF E.pdf 
[6] IANA.

[Online].

Available: https://www.iana.org/assignments/aaaparameters/aaaparameters.xml

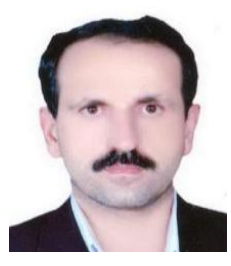

Saeed Mahdavi was born in 1966 in Iran Fasa. He graduated in Telecom University in 1996. He is specialized in switching system TDM. He had an international certificate from Alcatel, ZTE and Siemens. He had CCNA, CCNP and CCIE certificated. He is employee in TCF. He is an expert in NGN/IMS systems and having certificated KT Korea. $\mathrm{He}$ is a manager in telecom IMS project.

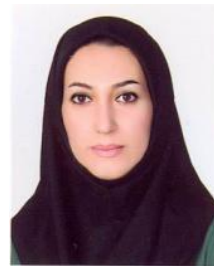

Maryam SabetSarvestani graduated from Amirkabir University of Technology (AUT) by the master degree in Information Technology Engineering - Computer Network. She had an international ZTE training NGN certificate and passed OJT IMS in TCF. Also she has CCNA and MCITP certificates.

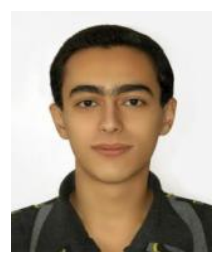

Mohammad Doosti graduated from Institute of Applied Science Technology, Jahad Deneshgahi (IASTJD), Shiraz, Iran, by the bachelor degree in Engineering Technology - Computer Networks. He had an international ZTE training NGN certificate and passed OJT IMS course in TCF. Also he is expert in CCNA, MCITP, MCSA, TMG and vSphere ESXI. 